entheses or increased inflammatory markers in the blood. The dominant leg was examined with US using a Logiq 9 (GE Medical, Milwaukee, WI, USA) with a ML 6-15 MHz transducer and a fixed pre-set with Doppler settings optimised for inflammatory flow. Both GS and Doppler examination were made.

The entheses were examined for hypoechogenicity, increased thickness, enthesophytes/ calcifications, erosions, and Doppler activity.

Results: No subjects had clinical signs of tendon or joint disease. Seven displayed various degrees of hypermobility and seven had various degrees of flatfoot; some in combination. None of the blood tests indicated any pathology.

On US erosions were only seen in one Achilles insertion (not shown in table). The Doppler activity was not measured in plantar fascia due to attenuation of the heel fad pad. All other US pathology present is seen in the table below.

Conclusions: Only minor pathological findings in the entheses of the lower limb were present in an age stratified cohort of healthy persons. The changes most frequently seen were bony changes in the insertion of the quadriceps and Achilles tendons. A weak tendency toward more pathological findings was seen in this cohort among men, compared to women, and additionally, with increasing age.

The findings suggest that US can be used to diagnose/examine subjects for pathological changes of the entheses although with caution regarding enthesophytes of the quadriceps and Achilles tendon.

[1] Terslev L et al. Defining enthesitis in spondyloarthritis by ultrasound: results of a Delphi process and of a reliability reading exercise. Arthritis Care Res 2014 May;66:741-8.

Disclosure of Interest: None declared

DOI: 10.1136/annrheumdis-2017-eular.3708

\section{FRI0655 ULTRASOUND FINDINGS OF FEEDING VESSELS AND BONE SURFACE IRREGULARITY IN WRIST JOINTS OF HEALTHY CONTROLS}

K. Misaki ${ }^{1}$, K. Ikeda ${ }^{2}$, K. Inoue ${ }^{1} .{ }^{1}$ Rheumatology, Kita-Harima Medical Center, Ono; ${ }^{2}$ Chiba University, Chiba, Japan

Background: Musculoskeletal ultrasound (MSKUS) is capable of visualizing synovitis and bone damage such as erosion. In particular, synovial vascularity as measured by power Doppler (PD) is correlated to rheumatoid arthritis disease activity, and PD signal reveals the prevalence of subclinical synovitis overlooked on physical examination. It is frequently difficult to distinguish bone erosion from normal concave surface of the bone. It is necessary for us to know these normal structures well in evaluating disease activity by using MSKUS. Here we examine the normal feeding vessels and bone surface irregularity in wrist joints.

Objectives: To clarify the distribution of feeding vessels in wrist joints and lunate surface irregularity of healthy controls by using MSKUS

Methods: The dorsal side of the bilateral wrists was scanned in 2 perpendicular planes with 2D-probe in healthy volunteers. The distribution and bilaterality of feeding vessels in the capsule and the extensor tendon sheath of wrist joint, and the evaluation of bone surface irregularity at lunate were validated.

Results: The distribution and bilaterality (Blt) of feeding vessels in healthy volunteers $(n=30)$ were as follows:

Above-trapezoid (Rt:100.0\%, Lt:100.0\%, Blt:100.0\%), intra-extensor digitorum tendon sheath (Rt:86.7\%, Lt:66.7\%, Blt:43.3\%), intra-extensor digiti minimi tendon sheath (Rt:30.0\%, Lt:30.0\%, Blt:10.0\%), above-capitate (Rt:23.3\%, Lt:30.0\%, Blt:10.0\%), above-triangular fibrocartilage complex (Rt:16.7\%, Lt:30.0\%, Blt:6.7\%), distal radial side of the radio-carpal joint (Rt:20.0\%, Lt:23.3\%, Blt:0.0\%), distal end of the ulna (Rt: $10.0 \%$, Lt: $16.7 \%$, Blt:0.0\%), dorsal side of the lunate (Rt:6.7\%, Lt:0.0\%, Blt:0.0\%), palmar side of the extensor digitorum tendon sheath (Rt:0.0\%, Lt:3.3\%, Blt:0.0\%). Feeding vessels from vascular channels were depicted at lunate (Rt:53.3\%, Lt:46.7\%, Blt:43.3\%), the distal side of the

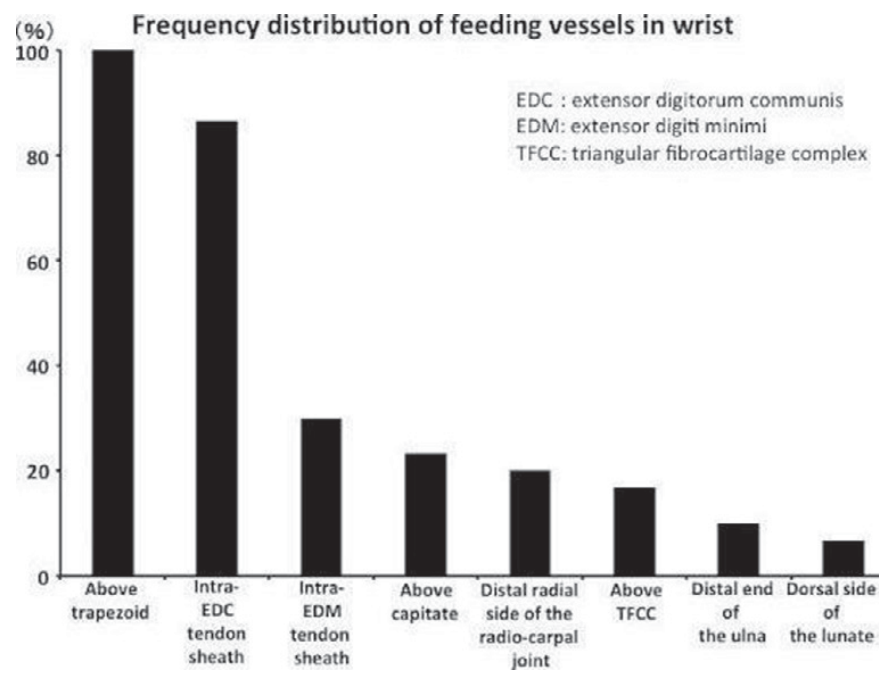

radius (Rt:20.0\%, Lt:16.7\%, Blt:3.3\%), triquetrum (Rt:10.0\%, Lt:16.7\%, Blt:0.0\%) and capitate (Rt:6.7\%, Lt:10.0\%, Blt:0.0\%). The frequency of the bone surface irregularity at lunate in healthy volunteers $(n=47)$ and the transverse diameter (TD) (Mean \pm S.D.) of those were Rt longitudinal plane:57.4\% (TD1.26 $\pm 0.33 \mathrm{~mm}$ ), Rt transverse plane: $51.1 \%$ (TD1.20 $\pm 0.31 \mathrm{~mm}$ ), Lt longitudinal plane: $68.1 \%$ (TD1.06 $\pm 0.28 \mathrm{~mm}$ ), and Lt transverse plane: $42.6 \%$ (TD1.29 $\pm 0.31 \mathrm{~mm})$. Bilateral evaluation of those was $53.2 \%$

Conclusions: MSKUS evaluation in wrist joints revealed various normal vessels and bone surface irregularity of lunate. It is necessary to distinguish a normal pattern from a pathological pattern in MSKUS examination.

Disclosure of Interest: None declared

DOI: 10.1136/annrheumdis-2017-eular.3745

\section{FRI0656 SEMIAUTOMATIC ANALYSIS OF EROSION VOLUME BY HR-PQCT IN PATIENTS WITH RHEUMATOID ARTHRITIS}

K. Chiba ${ }^{1}$, N. Iwamoto ${ }^{2}$, M. Osaki ${ }^{1}$, A. Kawakami ${ }^{2} .{ }^{1}$ Department of Orthopedic Surgery; ${ }^{2}$ Department of Rheumatology, Nagasaki University Graduate School of Biomedical Sciences, Nagasaki, Japan

Background: HR-pQCT is a high resolution CT dedicated to human extremities. It has been used mainly for osteoporosis, while in more recent years it has been applied to research in rheumatoid arthritis (RA).

Objectives: The purpose of this study is to develop a method to quantify the volume of erosions in RA patients semiautomatically using second generation HR-pQCT.

Methods: Twenty patients with RA ( $70 \pm 8$ years, 15 female, 5 male) participated in this study. The second and third MCP joints were scanned using HR-pQCT (XtremeCT II, Scanco Medical, Switzerland) at the voxel size of $61 \mu \mathrm{m}$.

The erosion volume was measured semiautomatically using the dedicated software (TRI/3D-BON, Ratoc System Engineering, Tokyo).

All concave regions on the bone surface around the MCP joints were extracted automatically by subtracting the bone region from the smoothed bone model. Erosions were selected manually by a medical doctor based on certain criteria. The volume of the erosions were measured by voxel counting.

Results: In a total of 37 joints (3 joints were excluded due to severe deformities), 40 erosions were detected by HR-pQCT (phalanx side: 9 erosions, metacarpal side: 31 erosions). The average volume of the erosions was $1.84 \mathrm{~mm}^{3}$, minimum $0.08 \mathrm{~mm}^{3}$, and maximum $16.3 \mathrm{~mm}^{3}$.

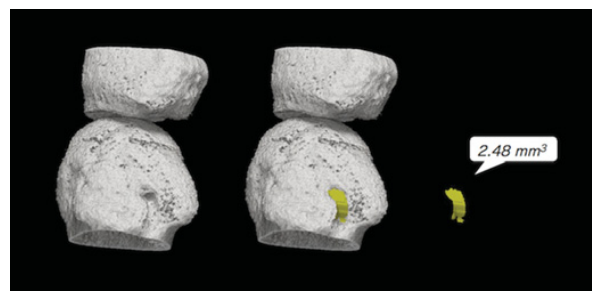

Conclusions: The semiautomatic method to quantify the volume of erosions at MCP joints in RA patients by HR-pQCT was developed.

The opinion as to whether concave regions are pathological erosion or physiological concave, vascular channel or recess of osteophyte is occasionally difficult and should be performed by an experienced tester.

Disclosure of Interest: None declared

DOI: 10.1136/annrheumdis-2017-eular.4144

\section{FRI0657 COMPARATIVE STUDY OF PAROTID ULTRASONOGRAPHY AND PAROTID GLAND ECT IN PREDICTING THE PATHOLOGICAL BIOPSY OF THE SALIVARY GLAND IN PATIENTS WITH PRIMARY SJÖGREN'S SYNDROME}

C. Xiaomei, L. Cong, W. Lijun, L. Cainan, S. Yamei. Department of Rheumatology and Immunology, People's Hospital of Xinjiang Uyghur Autonomous Region, urumqi, China

Objectives: To investigate the value of Parotid Ultrasonography and parotid gland radionuclide imaging in predicting salivary gland biopsy of primary Sjögren's syndrome (pSS)

Methods: Sixty - five patients with primary Sjogren 's syndrome and 38 patients with non - Sjogren's syndrome were enrolled in the Department of Rheumatology and Immunology, Xinjiang Uygur Autonomous Region People 's Hospital from October, 2015 to June, 2016. All patients were accpted the check of Parotid Ultrasonography, Parotid Gland ECT and labial gland biopsy. The data were analyzed statistically.

Results: The sensitivity and specificity of Parotid Ultrasonography in pSS were $65 \%$ and $76 \%$, respectively. The area under the ROC curve was $0.936 \pm 0.024$ for the diagnosis of pSS. The positive predictive rate of parotid gland ultrasonography was $84.0 \%$ and the negative predictive rate was $94.4 \%$. Parotid scintigraphy in pSS in the sensitivity of $80.0 \%$, specificity of $69.1 \%$. The positive predictive rate of parotid gland radionuclide imaging for labial gland biopsy was $78.9 \%$ and the negative predictive rate was $87.5 \%$. The diagnostic value of Parotid 